\title{
Low-power 2.4-GHz RF transceiver for wireless EEG module plug-and-play
}

\author{
J. P. Carmo \\ Polytechnic Institute of Braganca \\ Campus Santa Apolonia \\ 5301-854 Braganca, Portugal \\ jcarmo@ipb.pt
}

\author{
N. Dias, P. M. Mendes, C. Couto, J. H. Correia \\ University of Minho, Dept. Industrial Electronics \\ Campus Azurem \\ 4800-058 Guimaraes, Portugal
}

\begin{abstract}
This paper presents the design and fabrication of a radio-frequency (RF) transceiver fabricated in a UMC RF 0.18 $\mu \mathrm{m}$ CMOS process. The RF transceiver was built to operate at the $2.4 \mathrm{GHz}$ ISM band. The receiver has a sensibility of $-60 \mathrm{dBm}$ and consumes $6.3 \mathrm{~mW}$ from a $1.8 \mathrm{~V}$ supply. The transmitter delivers an output power of $0 \mathrm{dBm}$ with a power consumption of $11.2 \mathrm{~mW}$. The application is a wireless wearable electroencephalogram (EEG) braincap. Wireless EEG allows patients to wear the brain cap and maintain their mobility while simultaneously having their electrical brain activity monitored. A solution of an individual EEG module composed by an electrode, processing electronics and an antenna, allows a plug-and-play electrodes solution.
\end{abstract}

\section{INTRODUCTION}

An emerging field is the wireless monitoring of humanbody information. Body area network (BAN) comprises smart sensors able to communicate wirelessly to a base station. A wireless electroencephalogram (EEG) will provide a breakthrough in the monitoring, diagnostics and treatment of patients with neural diseases as epilepsy [1]. A wireless EEG module composed by the neural electrodes (noninvasive and distributed in a braincap), processing electronics and a RF transceiver with an associated antenna, will be an important breakthrough in EEG diagnostic (see Figure 1).

The RF transceiver (attached to an antenna) makes possible the data communication between the braincap and the external monitoring instrumentation used by health professionals. The transceiver must have low-power consumption, low-voltage supply and small-size so it can be assembled with an antenna and the EEG electrode. The modules are fed by a coin-sized battery placed in the braincap. The size-reduction achieved with these modules, make them suitable to be easily plugged and unplugged in the wireless EEG braincap according the interest of the medical doctors.

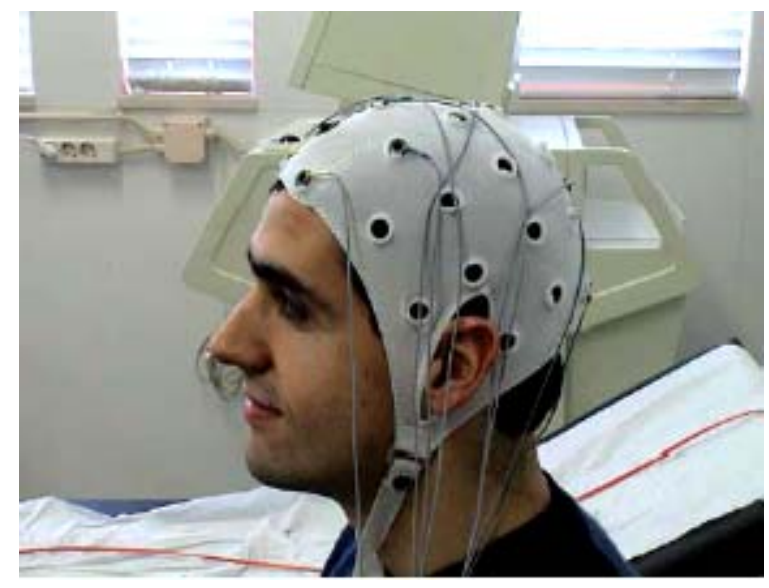

Figure 1. Classic EEG braincap with $\mathrm{Ag} / \mathrm{AgCl}$ electrodes and respective wires.

\section{RF CMOS TRANSCEIVER DESIGN}

The UMC RF $0.18 \mu \mathrm{m}$ CMOS process was used for the fabrication of a $2.4-\mathrm{GHz}$ RF transceiver. This process has a poly layer and six metal layers, allowing integrated spiral inductors (with a reasonable quality factor), high resistor values (a special layer is available) and a low-power supply of $1.8 \mathrm{~V}$. Therefore, a high on-chip integration is possible, in favour of better repeatability as well as less pin count [2].

Figure 2 shows the architecture of the transceiver, which consists of a receiver, a transmitter, and a frequency synthesizer. The receiver adopts a direct demodulation, by means of envelope detection. It is enough to achieve a bit error probability less that $10^{-6}$ with a sensibility of $-60 \mathrm{dBm}$, in a transmitted power of $0 \mathrm{dBm}$ using ASK modulation. These specifications makes the transceiver suitable to the operation in EEG environments, where the radiated power levels must be lowest. 


\section{A. Receiver}

Figure 3 shows the receiver's front-end schematic. The low-noise amplifier (LNA) is the first gain stage in the receiver path. In a LNA, the signal must be amplified as much as possible, with a small signal-to-noise ratio (SNR) decrease. This is achieved with the best noise figure (NF).

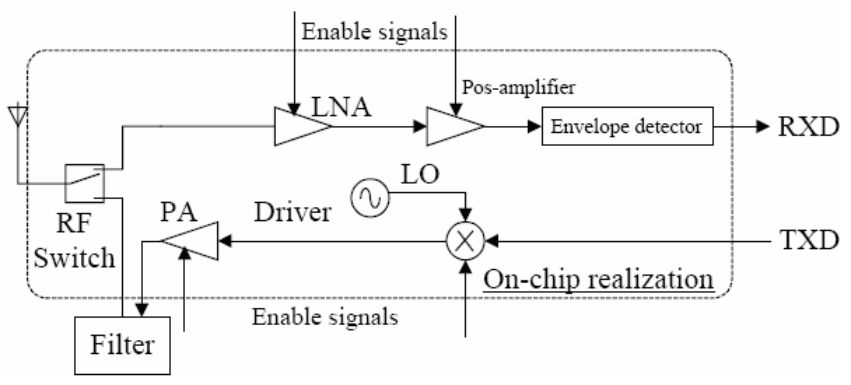

Figure 2. The block schematic of the transceiver.

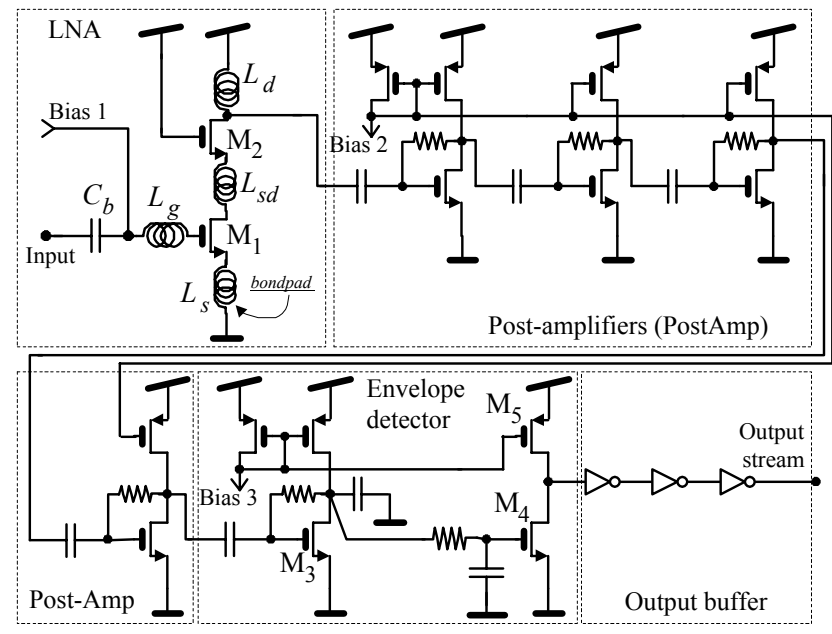

a)

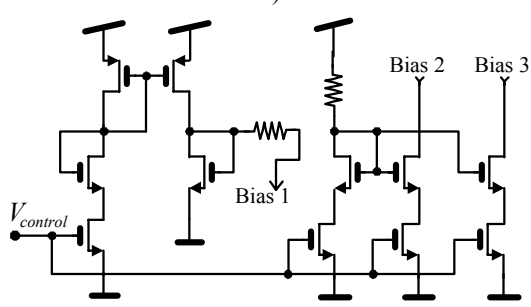

b)

Figure 3. The schematics of the a) receiver; b) bias and control circuitry.

The LNA is an inductively degenerated common source amplifier [3]. This makes the input impedance at $2.4 \mathrm{GHz}$ equal to $50 \Omega$, for matching with antenna-switch. Cascoding transistor $\mathrm{M}_{2}$ is used to increase the gain, to better isolate the output from input and to reduce the effect of $\mathrm{M}_{1}$ 's $C_{g s}$. The LNA is putted in the sleeping mode, by cutting the current in the polarization stage. The same principle applies to the all subsystems of the transceiver. The inductance $L_{\mathrm{s}}$ is implemented with the bonding connection to the external
PCB, which has been calculated to be $0.9 \mathrm{nH} / \mathrm{mm}$ [4]. The wires used to connect the die to an external PCB, with a RF substrate, has an inductance that adds to the LNA circuit. The use of the inductance $L_{s d}$, helps to reduce these effects.

A minimum RF level at the envelope detector is achieved, by means of further amplification of the signal at the LNA output. This minimum level defines the receiver's sensibility. Basically, the idea of the envelope detector is as follows: an increasing in the input amplifier implies a decrease in the $\mathrm{M}_{3}$ gate voltage (this keeps the branch current constant), meaning a decrease in the $\mathrm{M}_{4}$ 's gate voltage (after filtering), thus decreasing the transistor $\mathrm{M}_{4}$ current itself. When this current reaches a point that cancels with the transistor $\mathrm{M}_{5}$ mirror current, then the output capacitance starts to discharge and the output voltage goes to high.

\section{B. Transmitter}

The ASK modulated signal is generated by means of a switched power amplifier. The power amplifier has a cascade of five inverter, in order to drive the ASK output signal to the input of the power amplifier. The Figure 4 shows the schematic of the power amplifier, as well as, the whole transmitter. The network $L_{1}-C_{1}$ is tuned to the carrier frequency, while the emissions outside of the $2.4 \mathrm{GHz}$ band are reduced by the network $L_{2}-C_{2}$.

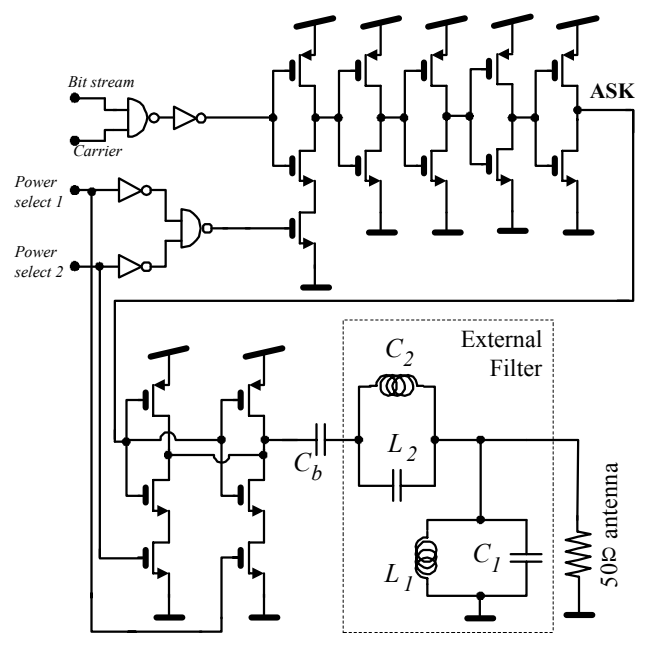

Figure 4. The schematic of the transmitter.

\section{Frequency synthesizer}

As depicted in Figure 5a), the PLL has a reference generator circuit with a crystal based oscillator at $20 \mathrm{MHz}$, followed by a phase-frequency difference circuit (PFD) without dead zone, a current steering charge pump (CP) and a third order passive filter. The passive section output is connected to the $\mathrm{VCO}$, that generates the desired frequency of $2.4 \mathrm{GHz}$. This frequency must be divided by 120 and connected to the PFD again, closing the loop.

In real PFDs there is an offset around the zero phase difference, and a gain inversion region takes place for phase 
differences higher than $2 \pi-\Delta \mathrm{rad}$. In this gain inversion region, the PFD outputs the wrong control signals increasing the phase and frequency differences between the inputs, and the lock time takes a sudden turn for the worse [5]. The implemented PFD has a linear gain in the range $-\pi$ thru $+\pi$, and a large constant gain in the range $[-2 \pi,-\pi]$ and $[+\pi,+2 \pi]$ [6]. This type of PFDs makes PLLs faster, compared to those using conventional PFDs.

The charge pump (CP) is a current steering type. This circuit avoids the conventional problem in CPs, that limits the opening and closing of current sources, in fact, in spite of being switched, the current is routing from the load to an alternative path, and from that path to the load.

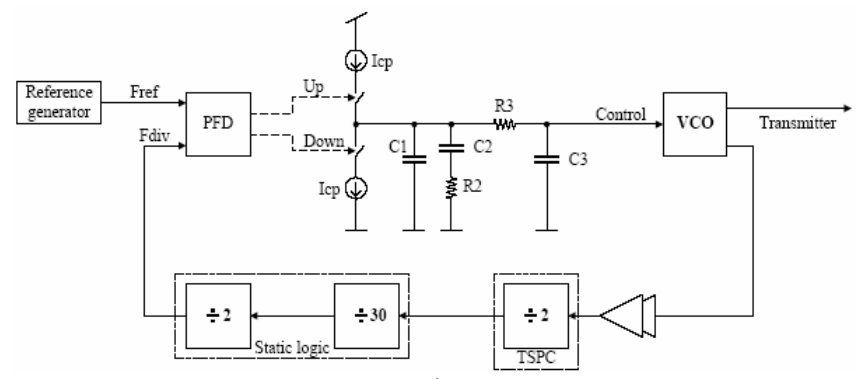

a)

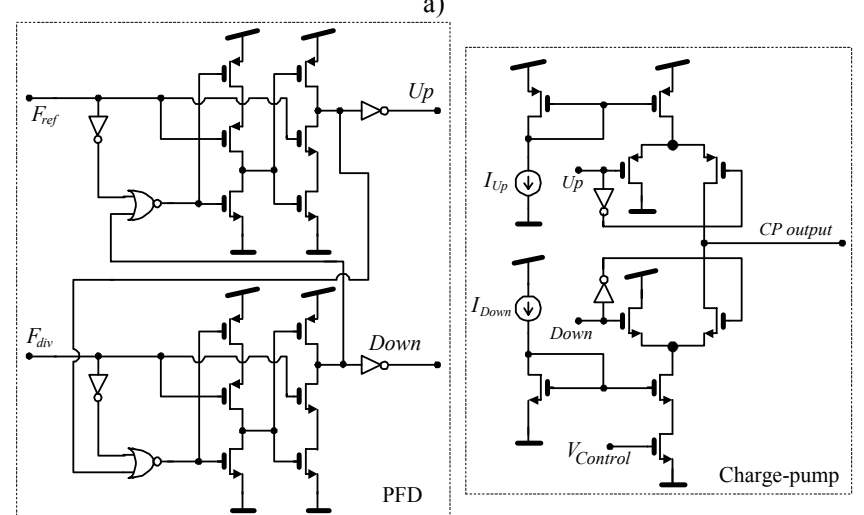

b)

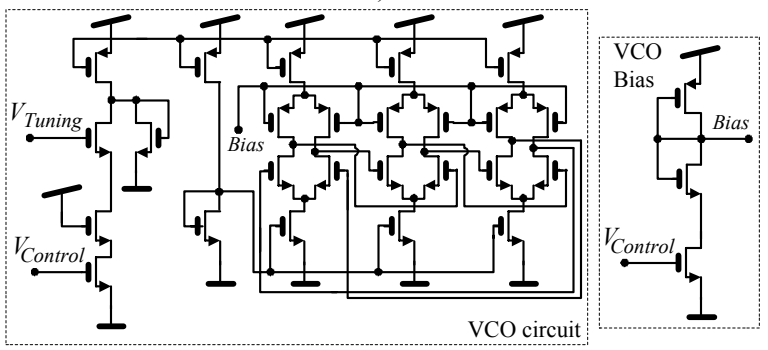

c)

Figure 5. a) The PLL structure, b) the schematic of the PFD-CP, and c) the schematic of the VCO.

A current starved ring oscillator was used as voltage controlled oscillator (VCO). Ring oscillators have more phase noise than LC oscillators. For overcoming this limitation, the bandwidth of the PLL must be high enough to "clean-up" the output spectrum around $2.4 \mathrm{GHz}$. A third order passive filter, composed by a second order section $\left(C_{1}\right.$, $C_{2}$ and $\left.R_{2}\right)$ and a first order section $\left(C_{3}\right.$ and $\left.R_{3}\right)$, providing an additional pole it is used. The first order filter reduces spurs caused by the multiples of reference frequency, whose consequence is the increasing of the phase noise at the output. The stability is guaranteed by putting this last pole five times above the PLL bandwidth and below the reference. A bandwidth of approximately two times the difference between the maximum and minimum frequencies generated by the VCO was used. The stability in the loop is obtained with a phase margin of $\pi / 4 \mathrm{rad}$.

The division by 120 in the feedback path is done with a cascade constituted by one half divider implemented with a true single phase clock (TSPC) logic [7], one divider by 30 , followed by a toggle flip-flop to ensure a duty-cycle of $50 \%$ at the PFD input. The TSPC logic was used to overcome the impossibility to implement the first toggle flip-flop with static logic in this technology. It is required a rail-to-rail input to work properly. The ratio of 30 was achieved with the use of simple frequency dividers by $2 / 3$ with modulus control.

\section{The antenna-switch}

The receiver or transmitter subsystems are connected to the antenna, by means of a digitally controlled antennaswitch. The isolation between non-connected ports must be high, keeping lowest the losses between connected ports. For a compact RF front-end the integration of the antenna-switch must in the same die of the transceiver (Figure 6).

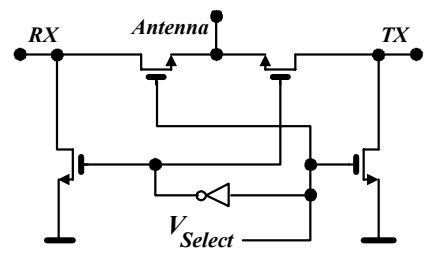

Figure 6. The antenna-switch.

\section{EXPERIMENTAL RESULTS}

Figure 7 shows the photograph of the RF CMOS transceiver die, which occupies an area of $1.5 \times 1.5 \mathrm{~mm}^{2}$, but the final structure can be optimized for including processing electronics for the acquired neural signals.

The experimental tests made to the transceiver, shown a total power consumption of $6.3 \mathrm{~mW}$ for the receiver $(4 \mathrm{~mW}$ for the LNA and $2.3 \mathrm{~mW}$ for the post-amplifier chain to the envelope detector), and $11.2 \mathrm{~mW}$ for the transmitter. The transmitter delivers a maximum output power of $1.28 \mathrm{~mW}$ (very close to the specified $0 \mathrm{dBm}$ ) with a power consumption of $11.2 \mathrm{~mW}$.

The LNA has a gain of $15.1 \mathrm{~dB}$, a NF of $1.076 \mathrm{~dB}$ and a $1 \mathrm{~dB}$ compression point of $-12.4 \mathrm{dBm}$. The LNA has also the stabilization factor $K=1.4$ (grater than the unity), that makes this amplifier unconditionally stable. The CP has Up and Down currents of $I_{U p}=173 \mu \mathrm{A}$ and $I_{\text {Down }}=178 \mu \mathrm{A}$, respectively, and with a detector constant gain $K_{\phi}=175$ $\mu \mathrm{A} / 2 \pi \mathrm{rad}$. The used VCO has the advantage to control the 
full range $[0,1.8 \mathrm{~V}]$, providing a frequency range of [2.016, $2.757 \mathrm{GHz}]$, with a tuning constant $K_{\mathrm{VCO}}=876.6 \mathrm{MHz} / \mathrm{V}$. The observations shown a time to lock of only $2.5 \mu \mathrm{s}$.

The antenna-switch has a maximum NF of $1.39 \mathrm{~dB}$. This antenna-switch provides a minimum port-isolation of 41.5 $\mathrm{dB}$ and a maximum insertion loss of $1.3 \mathrm{~dB}$, overcoming the reference values [8]. It was selected a commercial Impexa gigaAnt antenna, measuring $6.1 \times 3.1 \times 1 \mathrm{~mm}$ and weighting $0.05 \mathrm{~g}$ [9]. This antenna has a maximum return-loss of 2.5 , a bandwidth of $40 \mathrm{MHz}$, an efficiency of $55 \%$, and a nominal impedance of $50 \Omega$ at the $[2.4,2.5 \mathrm{GHz}]$ frequency range.

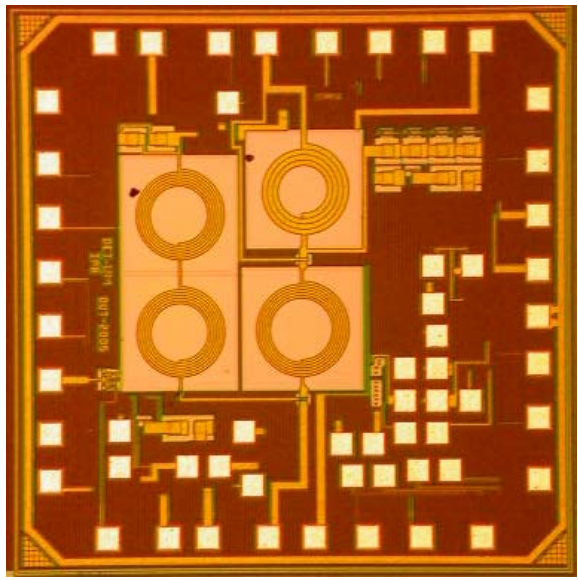

Figure 7. A die photograph of the RF CMOS transceiver.

\section{WIRELESS EEG MODULE}

The standard wireless EEG solutions use a braincap with wires running from the electrodes position to a bulky central unity (amplification, signal filtering and A-to-D conversion, RF transceiver and antenna) [1]. A more interesting solution is to use compact wireless EEG modules, where the electronics, the antenna and each electrode are mounted together. The power supply for these modules is obtained locally from a coin-sized battery placed in the braincap. Also, it is possible to integrate additional electronics (amplification, filtering and high-resolution digital conversion), for local signal processing in these small-size individual wireless EEG modules.

This solution fits the medical doctor requirements for an easy placement and removal of the electrodes in the braincap. Moreover, with this solution it is very easy to populate electrodes in different positions or takeoff. In many medical diagnostics the patients are monitorized only with a low number of electrodes (e.g. 2-5 electrodes), making possible to mount an EEG wireless-electrodes network with these plug-and-play modules. Figure 8 shows a 12-mm diameter $\mathrm{Ag} / \mathrm{AgCl} \mathrm{EEG}$ electrode for mounting the complete module. The RF CMOS transceiver and associated antenna will be mounted in the back of the electrode package.

\section{CONCLUSIONS}

A low-power RF transceiver for a wireless EEG single-electrode module was fabricated in a UMC RF CMOS
$0.18 \mu \mathrm{m}$ process. The transceiver consumes $6.3 \mathrm{~mW}$ in the receive mode and delivers $0 \mathrm{dBm}$ with a power consumption of $11.2 \mathrm{~mW}$ in the transmitting mode. These characteristics fulfill the requirements for short-range communications for using the $2.4 \mathrm{GHz}$ ISM band. It was also presented, a plugand-play EEG module composed by the fabricated RF transceiver, an associated antenna and an $\mathrm{Ag} / \mathrm{AgCl} \mathrm{EEG}$ electrode. The main goal is improving the EEG medical diagnostics and therapy by using devices which reduces healthcare costs and facilitates the diagnostic while at the same time preserving the mobility and lifestyle of patients.

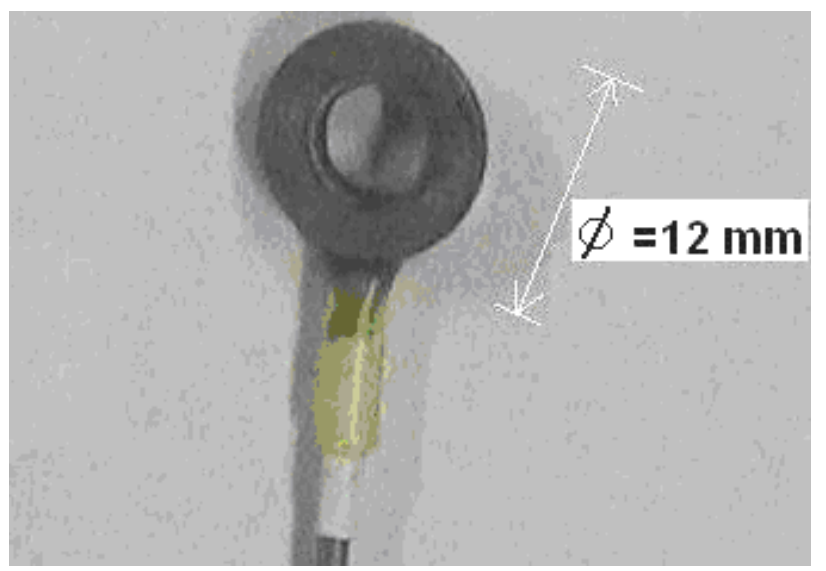

Figure 8. A photo of the $\mathrm{EEG} \mathrm{Ag} / \mathrm{AgCl}$ electrode $(12 \mathrm{~mm}$ of diameter) that will be used to mount the antenna with the RF CMOS transceiver on its back.

\section{REFERENCES}

[1] IMEC press releases, "Ambulatory EEG", Human ++ EU project, pp. 1-2, 2003, available at IMEC's web site: http://www.imec.be/human/.

[2] P. Choi, H. C. Park, S. Kim, S. Park, I. Nam, T. W. Kim, S. Park, S. Shin, M. S. Kim, K. Kang, Y. Ku, H. Choi, S. M. Park, K. Lee, “An experimental coin-sized radio for extremely low-power WPAN (IEEE 802.15.4) applications at 2.4 GHz", IEEE Journal of Solid-State Circuits, Vol. 38, Nr. 12, pp. 2258-2268, December 2003.

[3] D. Shaeffer, T. Lee, "A 1.5-V, 1.5-GHz CMOS low-noise amplifier", IEEE Journal of Solid-State Circuits, Vol. 39, Nr. 4, pp. 569-576, April 2004.

[4] F. Alimenti, P. Mezzanotte, L. Roselli, R. Sorrentino, "Modeling and characterization of the bonding-wire interconnection", IEEE Transactions on Microwave and Techniques, Vol. 49, Nr. 1, pp. 142150, January 2001

[5] K. Lee, B. Park, H. Lee, M. Yoh, "Phase-frequency detectors for fast frequency acquisition in zero-dead-zone CPPLLs for mobile communication systems", Proc. of the $29^{\text {th }}$ European Solid-State Circuits Conference, 16-18, Estoril, Portugal, September 2003.

[6] B. Kim, L. Kim, "A 250-MHz-2-GHz wide-range delay-locked loop", IEEE Journal of Solid-State Circuits, Vol. 40, Nr. 6, pp. 13101321, June 2005.

[7] S. Pellerano, S. Levantino, C. Samori, A. Lacaita, "A $13.5 \mathrm{~mW} \mathrm{5-}$ $\mathrm{GHz}$ frequency sinthesizer with dynamic logic frequency divider", IEEE Journal of Solid-State Circuits, Vol. 39, Nr. 2, pp. 378-383, February 2004.

[8] M. Ugajin, A. Yamagashi, J. Kodare, M. Harada, T. Tsukahara, "A $1-\mathrm{V}$ CMOS SOI Bluetooth RF transceiver using LC-tuned and transistor-current-source folded circuits", IEEE Journal of Solid-State Circuits, Vol. 39, Nr. 4, pp. 745-759, May 1997.

[9] Impexa gigaAnt antenna datasheet, available at the gigaAnt Coorporation web site: http://www.gigaant.com. 
MAIN AUTHOR VITAE

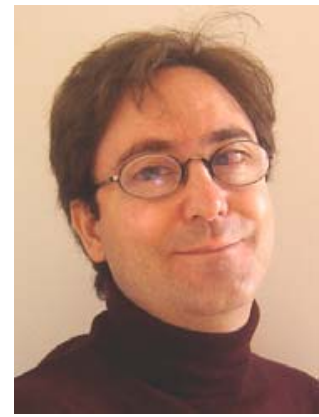

J.P. Carmo graduated in 1993 and received his MSc degree in 2002, both in Electrical Engineering and Computers from the University of Oporto, Oporto, Portugal. In 2007, he obtained the PhD degree in Industrial Electronics at the University of Minho, Portugal.

From 1999 to May 2008, he was a lecturer at the Polytechnic Institute of Bragança, Portugal.

Currently, he is currently involved and the leader in projects, concerning the research on RF applications and microsystems, at the Algoritmi center at University of Minho, Guimarães, Portugal. 